\title{
Phase transformation in ultra-thin films
}

\author{
J. Chakraborty \\ Materials Science and Technology Division \\ CSIR-National Metallurgical Laboratory \\ Jamshedpur-831 007, India \\ jay@nmlindia.org
}

Keywords: Phase transformation, thin films, film thickness, Gibbs free energy

\begin{abstract}
Thickness dependent structural phase transformation in thin polycrystalline metal films has been reviewed. Various effects of film thickness reduction on film microstructure have been identified. Film thickness dependent structural phase transformation has been treated thermodynamically taking polycrystalline titanium (Ti) thin film as model example.
\end{abstract}

\section{Introduction}

Thickness dependent structural phase transformation has been reported both in epitaxial and polycrystalline metallic thin films over the last decades [1-5]. Many metal films (Ni, Ti etc.) exhibits structural instability below certain critical film thickness where the film materials show metastable phases having crystal structures different than their equilibrium counterparts [1-5]. Sometime, the metastable structure coexists with the equilibrium phase in certain film thickness regime. Besides thin films, nanocrystalline metals prepared by high energy ball milling also exhibit polymorphic phase transformation below certain grain size. Lattice instability in metallic elements has been a special topic of recent review [6]. Various criteria for dynamic instability (such as phonon instability, elastic instability, total energy criterion etc.) of different lattice structure under external pressure and temperature have been theoretically outlined where electronic structure calculation plays an important role. However, general validity of these theoretical predictions is yet to be examined in case of lattice instability in nanocrystalline materials or in nanometer thick polycrystalline films. In the present contribution, effect of film thickness on the structural phase stability has been revisited in case of polycrystalline metallic thin films. It is known that structural phase transformation in stressed polycrystalline thin films is complicated by the presence of crystallites/grains of different orientations and possible crystallographic texture as compared to strained epitaxial thin films. At first, various consequences of thickness reduction in thin films will be discussed. Next, thermodynamic criteria for thickness dependent phase transformation in thin films will be outlined taking $h c p-f c c$ phase transformation in Ti thin film as a model example.

\section{Thickness dependence: polycrystalline thin films}

Majority of thin films are deposited by any physical or chemical vapor deposition process [7]. Film thickness can be changed by varying the deposition process parameters and deposition time. In polycrystalline thin films, variation of film thickness may lead to the change of microstructure of thin films which primarily involves the following: (i) change of crystallite size (or grain size) in thin films. Fig. 1(a) shows the change of crystallite size with film thickness reduction in $\mathrm{Zr}$ thin films [8]. Film thickness/crystallite size reduction further leads to change in the ratios of 'strain free' lattice parameters $\left(c_{o} / a_{o}\right)$ of $\mathrm{Zr}$ thin films [Fig. 1(b)]. In recent years lattice expansion has been observed in $\mathrm{Nb}$ thin films below 10nm crystallite size (Fig. 1(c)) [9] and a non-monotonic crystallite size dependence of the lattice parameters (i.e. both contraction and expansion of lattice) has been reported in ultra-thin nanocrystalline $\mathrm{Ni}$ thin films [10]. Such lattice contraction or lattice expansion arising due to certain stress fields (without going into the debate of the origin of stress existing in 
the literature) inside the grains leads to change of specific volume (volume/atom) of the lattice which may cause lattice instability due to the change in the total energy of the lattice structure [1, 6, 11]. Fig. 1(d) shows lattice expansion (i.e. increase of specific volume) in hexagonal close packed (hcp) Ti with decreasing film deposition time (i.e. decreasing film thickness) where the hcp $\mathrm{Ti}$ phase gradually transforms into face centered cubic $(f c c)$ Ti phase as crystallite size of $h c p \mathrm{Ti}$ decreases from $100 \mathrm{~nm}$ (for $25 \mathrm{~min}$. deposition time) to $10 \mathrm{~nm}$ (for $5 \mathrm{~min}$. deposition time) [4]. In this case, specific volume of $h c p$ Ti phase has been calculated from the strain free lattice parameters (determined from the diffraction stress analysis) of hcp Ti phase [4]. Specific volumes of $f c c \mathrm{Ti}$ phases are also shown in Fig. 1(d) for shorter film deposition period. It may be mentioned that based on elastic stability criterion, the possibility of a locally stable $f c c$ Ti phase was proposed earlier [11]. In the last section of the paper, stability of $h c p$ Ti phase in polycrystalline Ti thin films has been considered from a thermodynamic point of view.

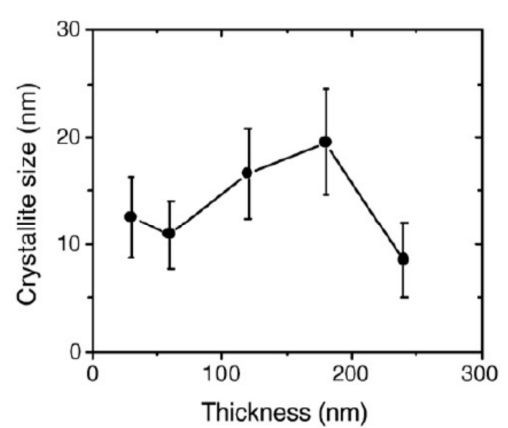

(a)

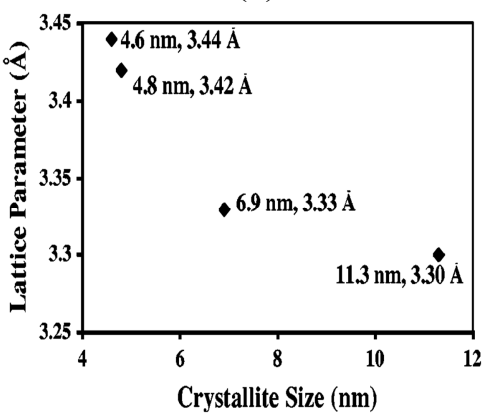

(c)

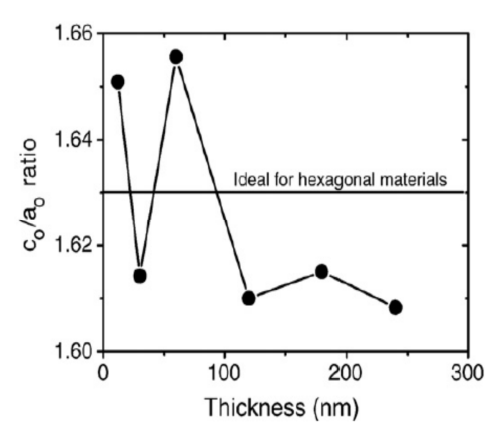

(b)

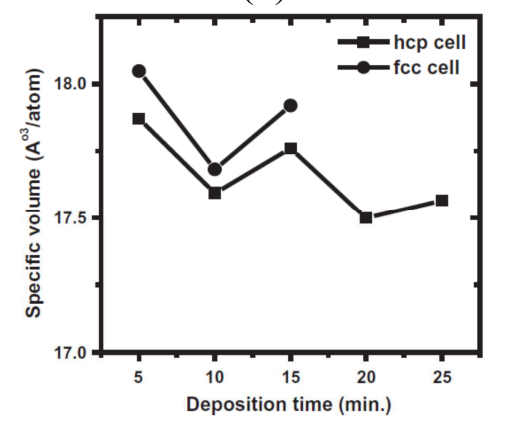

(d)

Figure 1: (a) Change of crystallite size with film thickness in Zr thin films [8]; (b) Change of strain free lattice parameters ratios $\left(c_{o} / a_{o}\right)$ in $\mathrm{Zr}$ thin films with film thickness [8]; (c) Lattice expansion in $\mathrm{Nb}$ thin films with decreasing crystallite size [9]; (d) Lattice expansion of hcp titanium in Ti thin films with decreasing deposition time (or film thickness) [4].

(ii) change of film thickness changes defect densities (density of dislocations, grain boundaries etc.) in polycrystalline thin films. It is to note that polycrystalline thin films usually contain high dislocation density (up to $10^{18} \mathrm{~m}^{-2}$ ) [12]. Accommodation of lattice defects during film growth usually results in large elastic strain in the film which leads to intrinsic/growth stresses (in-plane) in polycrystalline thin films because the film is dimensionally constrained by the substrate. (iii) Change in the orientation distribution of the crystallites often leads to very strong and sharp crystallographic textures in thin films $[13,14]$. Fig. 2(a) shows thickness dependent texture development corresponding to $\{0002\} h c p$ and $\{111\} f c c$ phases of titanium in Ti thin films deposited for 5min. ( $\sim 144 \mathrm{~nm}$ thick film $)$ and $15 \mathrm{~min}$. ( $\sim 432 \mathrm{~nm}$ thick Ti films $)$ [4]. For both phases strength and sharpness of the out of-plane texture increases with increasing film thickness. It must be mentioned that crystallographic texture in the film determines macroscopic average elastic constants of the polycrystalline thin film which is used to calculate the film stress from film strain.

Therefore, the above microstructural changes due to changing film thickness actually determine the intrinsic/growth stresses (in-plane) in polycrystalline thin film. It is known that thin films can withstand very large amount of stresses ( $\sim$ a few GPa). Fig.2 (b) \& (c) show thickness dependent 
stresses in $\mathrm{Zr}$ and $\mathrm{Ti}$ thin films respectively [8, 4]. Ti thin films undergoes $h c p$ to $f c c$ phase transformation (as film deposition time decreases) and the stress values in both the phases are shown in Fig. 2(c). Diffraction stress analysis by traditional $d$ - $\sin ^{2} \psi$ method [15] has been employed for determining the stresses in $\mathrm{Ti}$ and $\mathrm{Zr}$ thin films. In $\mathrm{Ti}$ thin films, $5 \mathrm{~min}$. deposition time corresponds to $144 \mathrm{~nm}$ thick film and $25 \mathrm{~min}$. deposition time corresponds to $720 \mathrm{~nm}$ thick film. For both $\mathrm{Ti}$ and $\mathrm{Zr}$ thin films, films stresses change from compressive to tensile with increasing film thickness. In the following section, change of film stress will be taken into account while considering the thermodynamics of structural phase stability in thin films.

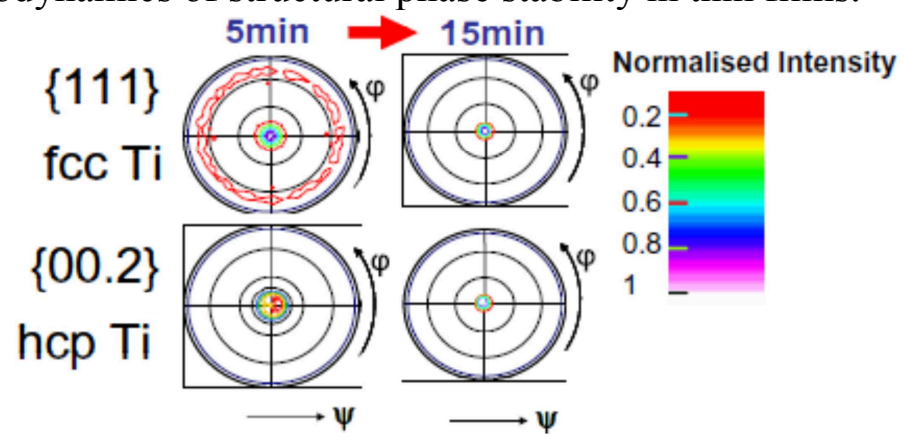

(a)

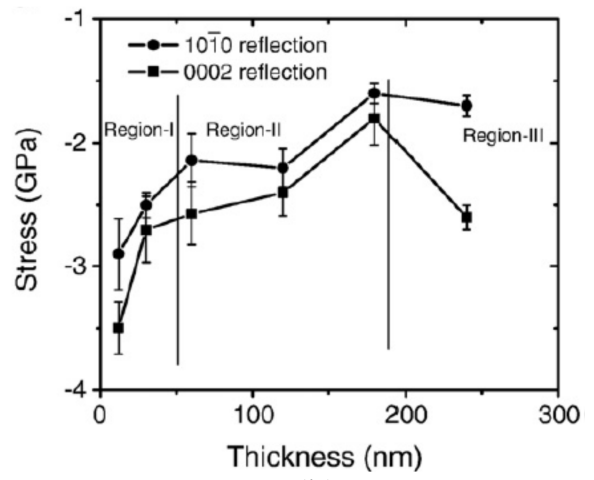

(b)

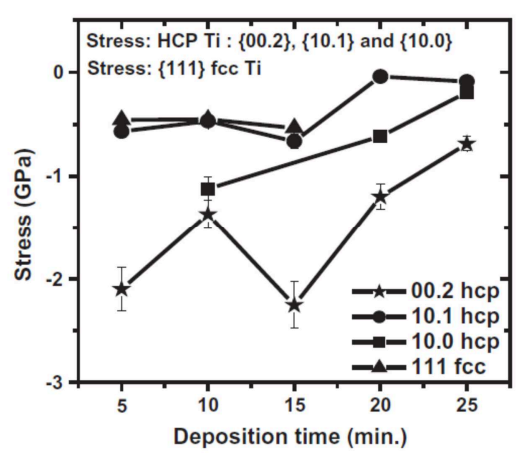

(c)

Figure 2: (a) Crystallographic texture evolution in $h c p$ and $f c c$ Ti phases in Ti thin films [4]; (b) Variation of intrinsic stress with changing film thickness in $\mathrm{Zr}$ thin films [8]; (c) Thickness dependent intrinsic stresses in $h c p$ and $f c c$ phases in Ti thin films [4]. Stress analysis has been done by measurement of orientation dependent inter-planar lattice spacing $(d)$ of individual peak reflections for both Ti and $\mathrm{Zr}$ films.

\section{Film thickness dependent structural stability: Thermodynamics}

Thermodynamically, for a given film thickness, structural stability of a phase in the film needs the total Gibbs free energy $(G)$ of the phase to be minimum. Any change of total free energy upon film thickness reduction can disturb this phase stability. As summarized in the last section, reduction of film thickness has two main consequences (i) reduction in crystallite size; (ii) change of film stresses. Both have large impact on the total Gibbs free energy of the film material. Total Gibbs free energy of the film is the sum of strain energy $\left(s^{\prime}\right)$, surface

energy $\left(\gamma^{\gamma}\right)$, interface energy $\left(\gamma^{/ /)}\right.$and bulk free energy $(\mu)$ of the material deposited as thin film. Changes of various energies upon film thickness reduction have been discussed below:

(i) Change in film stress due to film thickness reduction may result in the change of strain energy of the film. Strain energy can be calculated if the film stress and the biaxial elastic modulus of the thin film material are known; (ii) surface energy of the thin film is the energy of the interface between the film and the vacuum. Surface energy can be calculated from the energies of the unsaturated bonds present on the film surface since an atom on the film surface is not fully surrounded by other atoms. Hence, surface energy of thin polycrystalline films depends on the crystallographic orientation of the film surface (i.e. film texture). If there is a change of crystallographic texture of the film surface due to film thickness reduction, then it indicates change of the surface energy of the 
film. Furthermore, surface energy also increases as crystallite size decreases (due to film thickness reduction) since reduced crystallite size leads to higher surface to volume ratio of the crystallite. For example, during initial stage of film deposition (film thickness $<5 \mathrm{~nm}$ ), there are isolated atomic islands on the substrates where the surface energy becomes high as island size reduces. (iii) The interface energy is the energy of the film-substrate interface and also the grain boundary energy (which is typically $1 / 3^{\text {rd }}$ of the surface energy of the film) of the film. The film-substrate interface energy has basically two parts: (a) energy due to the chemical interaction between the film and substrate material; (b) energy due to the lattice mismatch between the film and the substrate. Change of interfacial energy may take place when the crystallite size reduces due to film thickness reduction and the reasons are: (i) change of lattice mismatch energy due to lattice expansion or contraction of the film with size reduction; (ii) change of grain boundary energy due to changing boundary width during lattice contraction or expansion with size reduction [9].

In the following, film thickness dependent stability of $h c p$ Ti phase in polycrystalline Ti thin films has been examined by thermodynamic calculations. It is reported that titanium undergoes $h c p$ to $f c c$ phase transformation in polycrystalline Ti thin films on Si (100) substrate below certain critical film thickness [4]. Bulk titanium has $h c p$ crystal structure at room temperature which is its equilibrium structure. Fcc titanium is a metastable phase and the difference between the bulk free energies of $f c c$ and $h c p \mathrm{Ti}\left(\mu_{f c c}-\mu_{h c p}\right)$ is $1.12 \times 10^{-20} \mathrm{~J} /$ atom $(0.07 \mathrm{eV} /$ atom $)$ [16] because the equilibrium crystal structure of Ti (i.e. $h c p$ ) in bulk has the lower bulk free energy. General expression of the total Gibbs free energy change during the phase transformation can be given as follows:

$\Delta G=\Delta \mu$ (bulk free energy change $)+\Delta s^{\prime}($ strain energy change $)+\Delta \gamma^{\prime}$ (Surface energy change $)+$ $\Delta \gamma^{\prime \prime}$ (interface energy change)

For polycrystalline $\mathrm{Ti}$ thin films on $\mathrm{Si}(100)$ substrate undergoing $f c c$ to hcp phase transformation, the total Gibbs free energy change of a volume element of single atomic column of Ti atoms perpendicular to the film-substrate interface can be written as follows [4]:

$$
\Delta G=-n \times 1.12 \times 10^{-20}+n \times\left[s_{h c p T i} \times \Omega_{h c p T i}-s_{f c c T i} \times \Omega_{f c c T i}\right]+\left(\frac{n}{t}\right)\left[\Omega_{h c p T i} \times \gamma_{h c p T i}-\Omega_{f c c T i} \gamma_{f c c T i}\right] .
$$

Where $n$ is the number of atoms in the atomic column and $t$ is the film thickness. $\Omega$ is the specific volume (volume/atom) of the respective Ti phases which can be calculated from the strain free lattice parameters of the respective Ti phases. Please note that the change of interfacial energy has been neglected in the above expression (equation (2)).

Second term on the right hand side of equation (2) is the change in the elastic strain energy during the transformation. The strain energy density $(s)$ of the respective Ti phase $(h c p$ and $f c c)$ is calculated from the expression $s=\sigma_{\|}^{2} / M$ where $\sigma_{\|}$is the rotationally symmetric biaxial stresses in respective Ti phase determined by diffraction stress analysis (see Fig.2(c)) for the stress values). Biaxial moduli $(M)$ of fiber textured $f c c(\{111\}$ textured) and $h c p(\{0002\}$ textured) phases of $\mathrm{Ti}$ have been calculated and they have been used. Fig. 3 shows the variation of strain energy densities for both phases of the Ti films at different deposition

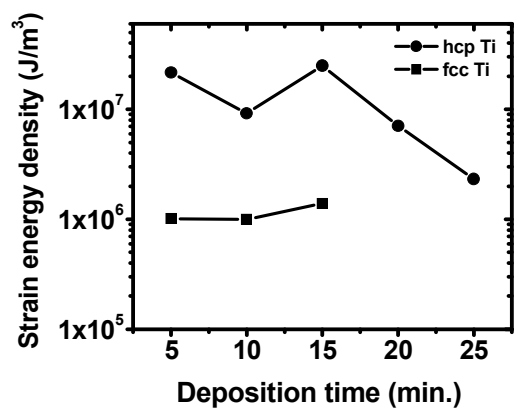

Figure 3: Variation of strain energy density with film deposition time [4]. 
times (i.e. for different Ti films thicknesses) [4]. The last term in equation (2) is the change in the surface free energy $(\gamma$ is the surface free energy per unit area) during phase transformation. Calculated surface free energies (per unit area) for $h c p$ Ti and $f c c$ Ti phases are $1.99 \mathrm{~J} / \mathrm{m}^{2}$ and $2.47 \mathrm{~J} / \mathrm{m}^{2}$ respectively (see ref. [4] for the detailed methodology for surface energy calculation).

For the $f c c$ to $h c p$ phase transformation to occur, the change of total Gibbs free energy has to be negative $(\Delta G<0)$ which is the condition of stable $h c p$ Ti phase. In the following calculation of free energy change $(\Delta G)$ has been done for $10 \mathrm{~min}$. film deposition time $(\sim 288 \mathrm{~nm}$ thick Ti film). The strain energy densities are $s_{h c p T i}=9.2 \times 10^{6} \mathrm{~J} / \mathrm{m}^{3} ; s_{f c c T i}=1 \times 10^{6} \mathrm{~J} / \mathrm{m}^{3}$ respectively from Fig.3. It is assumed that $\Omega_{h c p T i}=\Omega_{f c c T i}=17.5 \times 10^{-30} \mathrm{~m}^{3}$ (approximately). Taking the inter-planer lattice spacing (d) as $0.24019 \mathrm{~nm}$ in case of $\{111\}$ fiber textured $f c c$ Ti, for $288 \mathrm{~nm}$ thick film the number of atoms in the single atomic column can be calculated as 1199 . Thus the calculated values of change in strain energy is $17.2 \times 10^{-20} \mathrm{~J}$, the bulk free energy change is $-1343 \mathrm{~J}$ (negative) and the calculated values of change in surface free energy is $-3.5 \times 10^{-20} \mathrm{~J}$ (negative). Therefore the total change in Gibbs free energy can be calculated as follows:

$$
\Delta G=[-1343+17.2-3.5] \times 10^{-20} J=-1329.3 \times 10^{-20} J
$$

Hence $\Delta G$ becomes negative which indicates $f c c$ to $h c p$ transformation of $\mathrm{Ti}$ is possible in Ti thin films ( 288nm thick) deposited for $10 \mathrm{~min} . . \Delta G$ becomes more negative for longer deposition time (i.e. at higher film thickness) since the difference between strain energies of $h c p$ and $f c c$ Ti phases decreases with film deposition time (Fig.3) and the surface energy does not vary significantly with increasing film thickness and increasing film thickness. This indicates hcp Ti phase is more stable at higher film thickness which is indeed true for bulk Ti.

On the other hand, if the $f c c$ Ti phase has to be stabilized in $f c c$ to $h c p$ phase transformation, then the change of Gibbs free energy in equation (2) has to be positive $(\Delta G>0)$ which means:

$$
\left[s_{h c p T i} \times \Omega_{h c p T i}-s_{f c c T i} \times \Omega_{f c c T i}\right]+\left(\frac{1}{t}\right)\left[\Omega_{h c p T i} \times \gamma_{h c p T i}-\Omega_{f c c T i} \gamma_{f c c T i}\right]>1.12 \times 10^{-20} J
$$

After simplification and if $\Omega_{h c p T i}=\Omega_{f c c T i}=\Omega$ is assumed, condition in equation (3) can be written: $\quad\left[s_{\text {hcp Ti }}-s_{f c c T i}\right]+\left(\frac{1}{t}\right)\left[\gamma_{\text {hcp Ti }}-\gamma_{f c c T i}\right]>\frac{1.12 \times 10^{-20} J}{\Omega} \ldots \ldots \ldots . . .(4)$

It is clear that equation (4) can only be satisfied below certain critical film thickness (i.e. $t=t_{c}$ ) where both the conditions ' $s_{h c p T i} \gg s_{f c c T i}$ ' (trend is clear from Fig.3 as film deposition time reduces) and ' $\gamma_{h c p T i}>>\gamma_{f c c T i}$ ' (if surface energy of $h c p$ Ti becomes very large at reduced film thickness due to reduced size of Ti crystallites) are simultaneously satisfied. This is exactly the case of ultra-thin films (typically less than $30 \mathrm{~nm}$ or so) where it can be shown that both the conditions of surface and strain energies may get satisfied so that the left hand side of equation (4) can overcome the bulk free energy change on the right hand side. The above thermodynamic model can in principle be applied to examine the structural stability of a particular phase in a polycrystalline or epitaxial thin film.

\section{Summary}

Thickness dependent structural phase transformation in polycrystalline thin metallic films has been revisited. Various consequences of film thickness reduction on the crystal structure and microstructure of the film material have been identified. A thermodynamic model has been presented to study the thickness dependent $f c c-h c p$ phase transformation of $\mathrm{Ti}$ in polycrystalline $\mathrm{Ti}$ thin films which in principle can be applied to study the phase stability in other metallic thin films. 


\section{References}

[1] P. M. Marcus, F. Jona, J. Phys. Condens. Matter, 9 (1997) 6241-6246.

[2] P. Hemenger, H. Weik, Acta Cryst. 19 (1965) 690-691.

[3] D. Van Heerden, D. Josell and D. Shechtman, Acta Mater. 44 (1996) 297-306.

[4] J. Chakraborty, Kishor Kumar, Rajeev Ranjanm S. Ghosh Chowdhury and S. R. Singh, Acta Mater. 59 (2011) 2615-2623.

[5] Rajarshi Banerjee, Suliman A. Dregia, Hamish L. Fraser, Acta Mater. 47 (1999) 4225-4231.

[6] G. Grimvall, B. M-Kope, V. Ozolins, K. A. Persson, Reviews of Modern Physics, 84 (2012) 945-986.

[7] H. Windischmann, Crit. Rev. Solid State Mater. Sci. 17 (1992) 547.

[8] J. Chakraborty, Kishor Kumar, S. Mukherjee, S. K. Ray, Thin Solid Films, 516 (2008) 84798486.

[9] R. Banerjee, E. A. Sperling, G. B. Thompson, H. L. Fraser, S. Bose, P. Auyub, Appl. Phys. Lett.. 82 (2003) 4250-4252.

[10] J. Sheng, U. Welzel, E. J. Mittemeijer, Appl. Phys. Lett. , 97 (2010) 153109-1-153109-3.

[11] A. Aguayo, G. Murrieta, R. de. Coss, Phys. Rev. B 65 (2002) 092106.

[12] WD Nix, Metall Trans 20A (1989) 2217.

[13] CV Thompson, Annu. Rev. Mater. Sci., 20 (1990) 245.

[14] Carl. V. Thompson, Roland Carel, Mater. Sci. and Engg. B32 (1995) 211-219

[15] I. C. Noyan, J. B. Cohen Residual Stress, (Springer-Verlag New York Inc. 1987)

[16] AT Paxton, M. Methfessel, HM Polatogiou, Phys. Rev. B 41 (1990) 8127. 